\title{
RDDpred: a condition-specific RNA-editing prediction model from RNA-seq data
}

\author{
Min-su Kim ${ }^{1}$, Benjamin Hur ${ }^{1}$ and Sun Kim ${ }^{1,2,3^{*}}$ \\ From The Fourteenth Asia Pacific Bioinformatics Conference (APBC 2016) \\ San Francisco, CA, USA. 11 - 13 January 2016
}

\begin{abstract}
Background: RNA-editing is an important post-transcriptional RNA sequence modification performed by two catalytic enzymes, "ADAR"(A-to-I) and "APOBEC"(C-to-U). By utilizing high-throughput sequencing technologies, the biological function of RNA-editing has been actively investigated. Currently, RNA-editing is considered to be a key regulator that controls various cellular functions, such as protein activity, alternative splicing pattern of mRNA, and substitution of miRNA targeting site. DARNED, a public RDD database, reported that there are more than 300-thousands RNA-editing sites detected in human genome(hg19). Moreover, multiple studies suggested that RNA-editing events occur in highly specific conditions. According to DARNED, $97.62 \%$ of registered editing sites were detected in a single tissue or in a specific condition, which also supports that the RNA-editing events occur condition-specifically. Since RNA-seq can capture the whole landscape of transcriptome, RNA-seq is widely used for RDD prediction. However, significant amounts of false positives or artefacts can be generated when detecting RNA-editing from RNA-seq. Since it is difficult to perform experimental validation at the whole-transcriptome scale, there should be a powerful computational tool to distinguish true RNA-editing events from artefacts.

Result: We developed RDDpred, a Random Forest RDD classifier. RDDpred reports potentially true RNA-editing events from RNA-seq data. RDDpred was tested with two publicly available RNA-editing datasets and successfully reproduced RDDs reported in the two studies (90\%, $95 \%$ ) while rejecting false-discoveries (NPV: 75 \%, 84 \%).

Conclusion: RDDpred automatically compiles condition-specific training examples without experimental validations and then construct a RDD classifier. As far as we know, RDDpred is the very first machine-learning based automated pipeline for RDD prediction. We believe that RDDpred will be very useful and can contribute significantly to the study of condition-specific RNA-editing. RDDpred is available at http : / biohealth. snu . ac . kr/software / RDDpred.
\end{abstract}

Keywords: RNA-editing, Condition-specific, Machine-learning, Random forest, RNA-seq, Systematic artefact

\section{Background}

RNA-editing: a biologically crucial regulator and highly condition-specific event

RNA-editing event is defined as a post-transcriptional RNA sequence modification [1]. Currently, there are two known RNA-editing mechanisms, performed by two different catalytic enzymes, "ADAR" (A-to-I) and

\footnotetext{
*Correspondence: sunkim.bioinfo@snu.ac.kr

1 Interdisciplinary Program in Bioinformatics, Seoul National University, Seoul, Republic of Korea

${ }^{2}$ Department of Computer Science and Engineering, Seoul National

University, Seoul, Republic of Korea

Full list of author information is available at the end of the article
}

"APOBEC" (C-to-U) [2, 3]. The most common type of editing in metazoans is the one catalyzed by the ADAR family of enzymes [4]. By utilizing high-throughput sequencing technologies, the biological function of RNAediting has been actively investigated [5-7]. Currently, RNA-editing is considered to be a key regulator that controls various cellular functions including protein activity, alternative splicing pattern of mRNA and substitution of miRNA targeting site [1, 8-10].

Moreover, there are multiple studies that showed direct relation of RNA-editing to biological phenotypes. For example, Galeano's group showed that the 
editing events in glioblastoma by ADAR2 enzymes are crucial for pathogenesis and claimed that ADARclass enzyme can be considered as a tumor-suppressor [11]. And in APOBEC3G, a type of APOBEC-class enzyme causes HIV-1 retroviral inactivation by deamination [12].

DARNED, a well-curated public RNA-editing database have more than 300-thousands editing sites detected in the human genome hg19 [13]. Interestingly, the expression patterns of editing events in different conditions varied significantly. For example, in DARNED database, 333,164 editing sites in hg19 are registered from 21 independent studies in 139 tissues. The conservation rate among tissues is strikingly low, $97.62 \%$ of these registered sites were detected from a single tissue or condition (Fig. 1). Moreover, multiple studies suggest that RNA-editing events can be involved in condition-specific regulation of genetic functions [14, 15]. Taken together, it is reasonable to believe that RNA-editing is highly condition-specific event.

\section{RNA-seq: an important tool for investigating condition-specific RNA-editing patterns}

RNA-seq, a high-throughput sequencing of transcriptome, is a powerful method for investigating wholetranscriptome status. Since the nature of the technology is taking a snapshot of cells with massive sequencing reads, it is suitable for detecting condition-specific events in whole-transcriptome scale. Therefore, it is also suited for detecting RNA-editing events that have such conditionspecific characteristics. There have been a number of studies that used RNA-seq to reveal condition-specific editing patterns in whole-transcriptome scale [5-7].

\section{Systematic artefacts: the major huddle to detect authentic RNA-editing events from RNA-seq}

Even though RNA-seq is suitable for RNA-editing detection, it is also true that the current computational pipelines of RNA-editing detection with RNA-seq have considerable false-positive risks. In 2012 Nature Biotechnology journal, an article "The difficult calls in RNA editing", reports interviews with eight prominent RNA-editing researchers. They pointed out that false-positive calling is one of the most challenging problems in RNA-editing detection with RNA-seq [16].

The false-positives caused by mis-alignment of shortreads can be termed "Systematic Artefacts" due to their inherent and reproducible characteristics. Systematic artefacts can be caused by various reasons, (a) inherent duplications/repeats within genomic sequences, (b) ambiguity caused by splicing-junctions, (c) prevalent polymorphisms between individuals and (d) shortness of sequencing reads $[17,18]$. This inherent and reproducible error has been assumed to be one of the major confounding factors while detecting sequence variants $[19,20]$.

To assess the significance of confounding effects caused by systematic artefacts, we performed a simple simulation test in order to measure the false-detection rate of RNA-variants caused by mis-alignments. We used RNASTAR, a state-of-art alignment tool in base-accuracy [20, 21], to evaluate inherent risks of false-positive detection in the human genome hg19 (Fig. 2). We simulated 10-millions reads from mRNA-seq [22] with $1 \%$ simulated SNVs (513-thousand sites) and aligned them into the hg19 genome sequence. These $1 \%$ of simulated SNVs are for representing the individual genetic

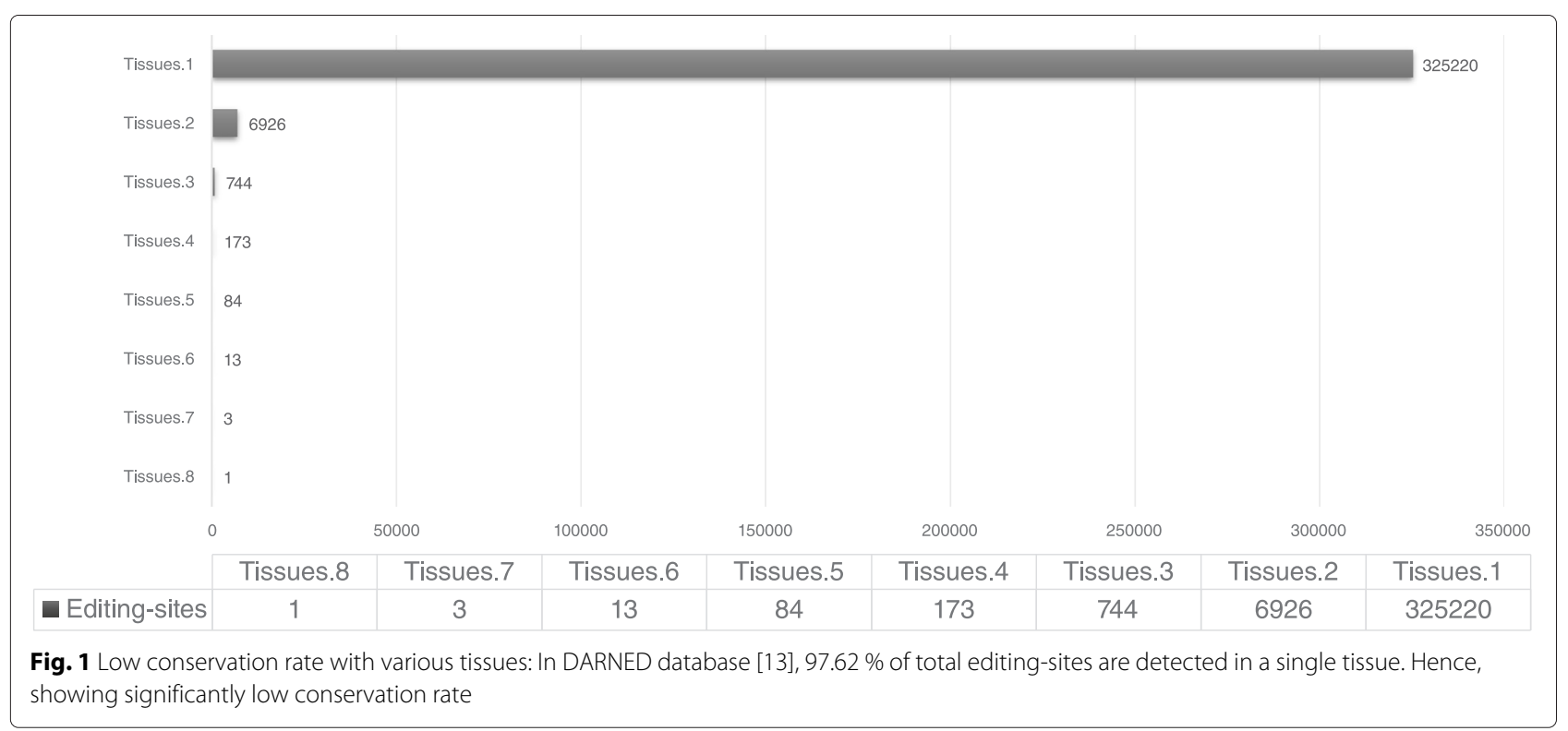




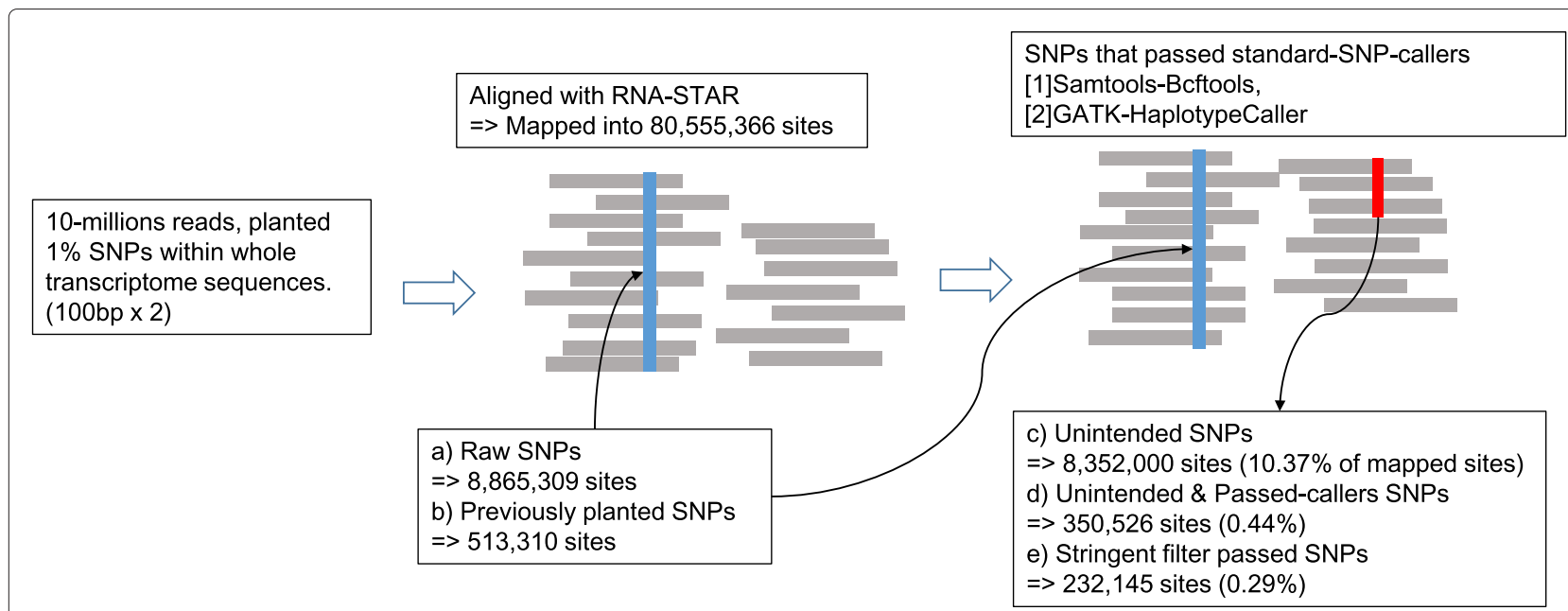

Fig. 2 Substantial amounts of systematic artefacts: As simulation-test results, significant amounts of artefacts that consist of $0.29 \%$ of total mapped sites are detected, which turned out to be indistinguishable even by the method combined with two state-of-art variant-callers and extensive $a$ priori knowledges of genomic repeats

differences including SNPs, somatic mutations, and RNA-editings.

We generated 10 simulated data and aligned them (Fig. 3). In addition to the planted sites, we discovered that average 8.3 millions SNVs were detected from each 10-million reads(while we have planted 513-thousands sites). This is clearly artefacts. Such considerable number of unintended artefacts consist of $10.37 \%$ of total mapped sites. Moreover, $2.78 \%$ (232-thousands sites) of these unintended artefacts cannot be excluded by standard SNP-callers $[23,24]$ or by stringent filtering with errorinducible regions in hg19 genome [25] (Fig. 2). The result suggests that if we use 10-millions reads to detect RNAediting, we will be confronted at least 232-thousands of artefacts that is difficult to be excluded by standard methods (Table 1 ).

\section{Three distinct computational approaches that addressed the artefact-issue}

To handle systematic artefacts in RNA-seq, a number of computational approaches have been developed. These can be categorized into three groups in terms of features they used: (a) A priori knowledge based filtering [26, 27], (b) Computational simulation of artefacts [6], (c) Machine-learning based prediction model [5, 28].

A priori knowledge based filtering used public genomic features, such as Alu repeats, genomic duplications, and pseudogenes, to assess the detected editing-sites directly. For instance, Li's group used public annotation of genomic repeats to filter out potential artefacts within the detected RDD(RNA/DNA Difference) sites [26]. On the other hand, the approach based on computational simulation of artefacts rather utilizes calculated features than public

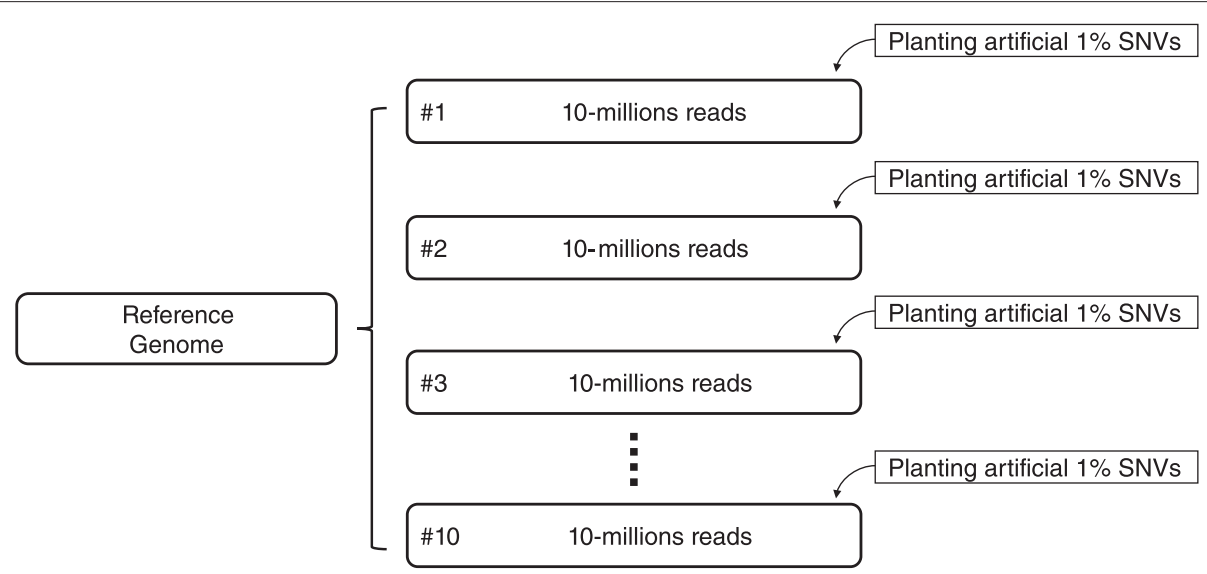

Fig. 3 Simulation dataset preparation: We generated 10 simulated sequencing reads from spliced genomes (spliced by NCBI refseq annotation). Note that each reads are generated with different sets of SNVs 
Table 1 Artefact simulation results: resulted from 10-times of iterations

\begin{tabular}{|c|c|c|c|c|c|c|}
\hline & Mapped reads & Mapped sites & Raw SNPs & Unintended SNPs & Caller passed & Filtered \\
\hline Trial.1 & $9,734,787$ & $80,552,288$ & $8,872,433$ & $8,358,426$ & 350,694 & 231,972 \\
\hline Trial.2 & $9,735,558$ & $80,558,479$ & $8,878,304$ & $8,365,007$ & 350,670 & 232,496 \\
\hline Trial.3 & $9,733,473$ & $80,568,898$ & $8,880,681$ & $8,366,553$ & 350,136 & 231,912 \\
\hline Trial.4 & $9,733,159$ & $80,570,416$ & $8,879,502$ & $8,365,311$ & 350,442 & 231,539 \\
\hline Trial. 5 & $9,733,939$ & $80,545,810$ & $8,853,408$ & $8,339,822$ & 350,332 & 232,008 \\
\hline Trial.6 & $9,733,507$ & $80,542,007$ & $8,838,870$ & $8,326,074$ & 350,917 & 232,649 \\
\hline Trial.7 & $9,734,222$ & $80,555,307$ & $8,859,741$ & $8,346,628$ & 350,390 & 232,128 \\
\hline Trial.8 & $9,735,046$ & $80,562,701$ & $8,874,369$ & $8,361,655$ & 350,807 & 232,063 \\
\hline Trial.9 & $9,733,971$ & $80,555,609$ & $8,852,720$ & $8,339,866$ & 350,059 & 232,336 \\
\hline Trial.10 & $9,734,717$ & $80,542,143$ & $8,863,065$ & $8,350,655$ & 350,809 & 232,347 \\
\hline Average & $9,734,238$ & $80,555,366$ & $8,865,309$ & $8,352,000$ & 350,526 & 232,145 \\
\hline
\end{tabular}

features. Peng's group used extensively simulated RNAseq to predict inherent error-inducible regions in genome sequence and used them as a filter [6].

Unlike the filter-based methods that directly assess RDD candidate sites with pre-defined filters, machinelearning based methods generates a predictor in advance. The predictor, or machine-learning classifier, is trained to learn the differences between true and false examples. As an example, Laurent's group generated a Random Forest predictor that utilizes read-alignment patterns as attributes. With 77 attributes, Laurent's group generated a predictor and demonstrated it has $87 \%$ of estimated accuracy by experimental validation [5]. As mentioned, since RNA-editing events are occurred highly condition-specifically, machine-learning approach might have an advantage in that they pursue more data-driven method by generating condition-specific model.

\section{Machine-learning based RNA-editing prediction became possible}

Laurent's work [5] was the first successful demonstration to show that a machine learning approach for RNAediting prediction is both feasible and sensitive. However, to be a general-purpose model, there are several limitations. First of all, a predictor needs a training data that consists of positive and negative examples. And in Laurent's study, they collected the both training examples from additionally performed Sanger-seq [5]. However, as we emphasized, RNA-editing is a condition specific event. And, since Laurent's approach used experimentally verified training examples specific to their own conditions, the model might not be applicable in different conditions unless additional sequencing is performed. Therefore, it is more cost-efficient if we can avoid the experimental validations with utilizing the machinelearning approach.

\section{Methods}

Here we introduce RDDpred, a software package that is generally usable and do not need an experimental validation to prepare condition-specific training examples. Hence, RDDpred prepares condition-specific training data directly from input sequencing data or raw RDD candidates. In order to collect positive examples without experimental validations, we utilized two wellorganized RNA-editing databases, RADAR and DARNED $[13,29]$. Since we consider systematic artefacts as major cause of false-positives, we collect negative examples by utilizing the MES method that calculates the errorinducible regions within genome during alignments [6]. After collecting positive/negative examples from input data, all the remaining sites are considered as targets for prediction. RDDpred is a Random Forest predictor that utilizes 15 features that reflect the read-alignment patterns. The overall prediction scheme is illustrated in Fig. 4.

\section{Implementation of RDDpred}

We tested RDDpred in Python (2.7.3), Samtools-Bcftools (1.2.1), WEKA (3.6.12) package, in linux environment.

\section{1) Input and output of RDDpred}

RDDpred takes alignment results as input data and gives the prediction results of each SNVs, or RDD candidates as outputs. The raw RDD candidates are detected with Samtools-Bcftools pipeline [23] while the prediction model is trained by using WEKA package [30].

\section{2) Selection of alignment tool by the user}

RDDpred can take inputs from any kind of alignment methods providing BAM-format outputs. However, we recommended RNA-STAR for its high degree of overall accuracy and ultra-fast performance $[20,21]$. 


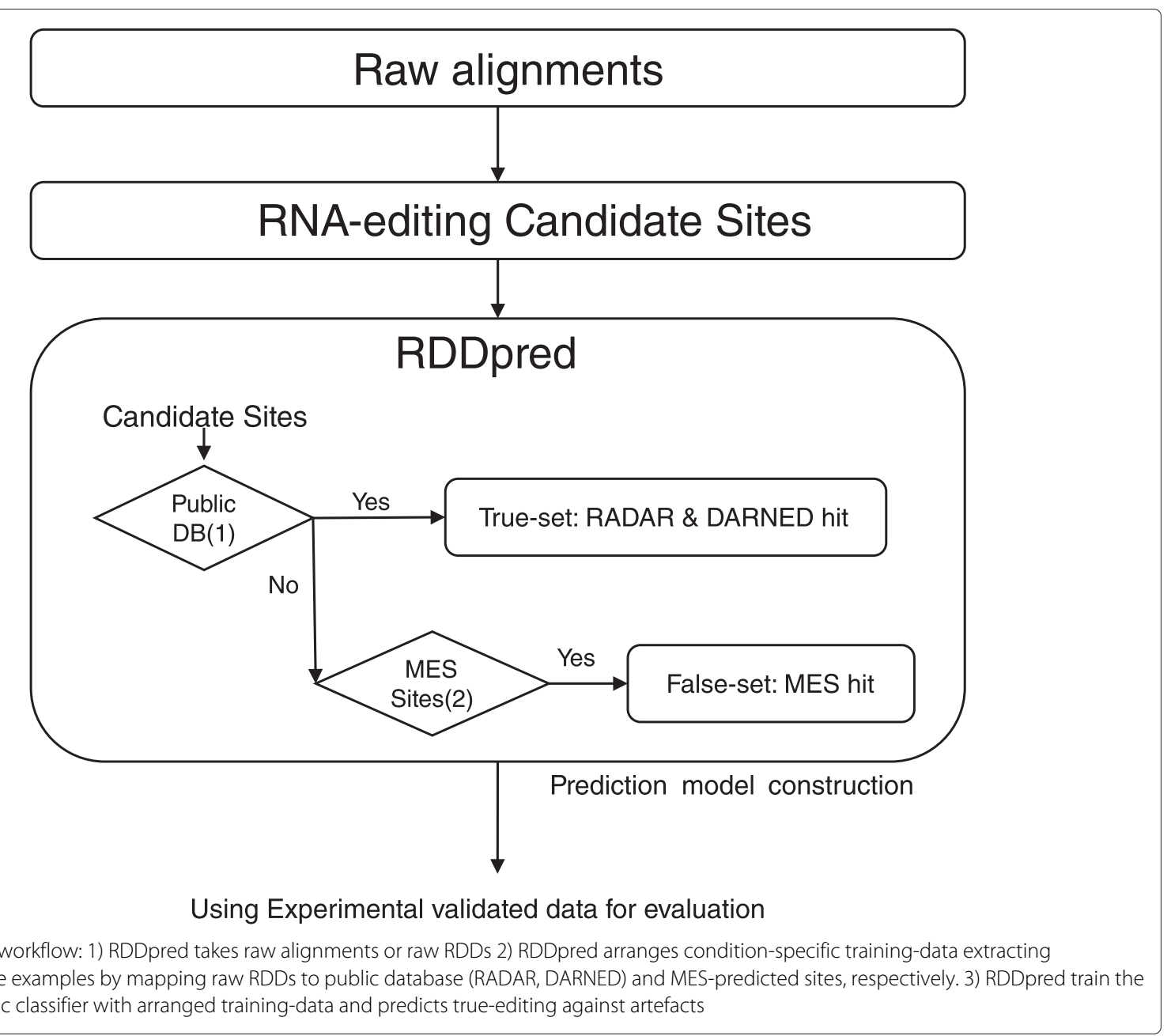

\section{Condition-specific training data preparation}

1) Positive-set of training data: utilizing public databases, RADAR and DARNED

RADAR and DARNED databases include 2.5 million, 300thousands of curated sites respectively [13, 29]. These two databases share a considerable portion of sites, 150thousands sites. Since the pre-known sites are already proved to have editing potential, we can use the sites matched to the consensus sites as positive examples (Fig. 4). Since RDDpred takes the positive sites as an input, users can change or supplement the sites that are considered as true events.

\section{2) Negative-set of training data: applying MES artefact calculation method}

To build a predictor, we also need negative examples. To address this issue, we utilized the MES method, a computational simulation for predicting error-inducible regions within genomes [6] (Fig. 5). With MES method, we can calculate error-inducible regions specific to the conditions, such as, SNPs combination of the samples, the experimental specification of sequencing, and the choice of alignment method. And if we calculate the error-inducible regions specific to our conditions, then we can consider them as potential error-prone regions. Therefore, just like collecting positive examples, we can reasonably assume the portion of RDD candidate sites that belong to those error-prone regions are probably systematic artefacts, thus using them as negative examples (Fig. 4).

\section{RDDpred predictor description}

\section{1) RDDpred mainly focuses on systematic artefacts}

It is known that there are various types of artefacts from RNA-seq, such as amplification errors during library construction, sequencing errors, and errors by mis-alignments. Unlike former events, errors by misalignments shows different characteristics that they are more reproducible. Since the errors from library construction and sequencing procedures are transient in general, they can be excluded by replicating experiments. On the other hand, since the errors by mis-alignments, 


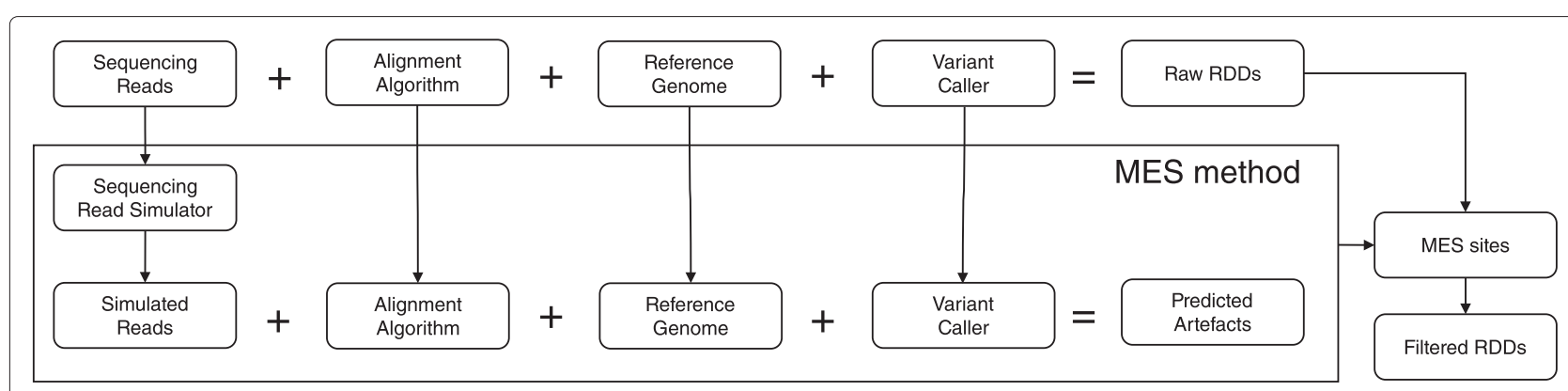

Fig. 5 MES-calculation workflow: 1) MES method simulated randomly mutated sequencing reads. 2) Then, aligns them into genome sequences with an alignment tool of interest. 3) After the alignment finishes, uses variant-caller to detect raw SNPs. 4) Picks the SNPs sites that was not planted originally, i.e., unintended artefacts

or systematic artefacts, are inherent to specific alignment method, they might not be excluded even after multiple replications. Therefore, RDDpred mainly focuses on detecting systematic artefacts with considering other artefacts as well.

\section{2) Read-alignment pattern: a valuable source for distinguishing systematic artefacts}

Read-alignment pattern is defined as local status of alignments in variant sites that has been utilized to distinguish artefacts [23]. There are at least six distinct categories of attributes calculated from read-alignments pattern. RDDpred utilizes 15 attributes of the six categories to generate a predictor, which are listed in Table 2. The six categories, such as "Read Depth", "Allele Segregation", "Mapping Quality", "Read Position", "Base Quality", and "Read strand" are known to have significant prediction power and also utilized by Samtools-Bcftools pipeline [23]. The attributes are basically measured as statistics to distinguish how the reads with variants are different with the reads without variants. RDDpred observed massive examples of positive/negative sites to learn how the statistics are represented differently between them. All of 15 attributes are calculated with Samtools-Bcftools pipeline during raw RDD detection [23]. Also we used WEKA, a data mining package, to train a prediction model [30]. Among the algorithms supported in WEKA, we chose Random-Forest algorithm, which showed the best performance in our evaluation datasets and showed significant performance in Laurent's study [5].

\section{3) The 15 attributes for RDDpred}

As mentioned, the 15 attributes are categorized into six category, (a) "Read Depth" category represents read-count in editing sites. (b) "Allele Segregation" category includes four attributes, such as VAF, SGB, FQ, and CallQual, respectively. All of these attributes are calculated from edited read-ratio against total reads. (c) "Mapping Quality" category of attributes reflects how the alignments of reads are well-performed, which utilizes alignment scores that the aligner generates. Four attributes, such as PV3, MQB, MQ0F and MQ belongs to this category. (d) "Read Position" category includes three attributes, such as VDB, RPB, and PV4, which represent how the positions of variants are biased within sequencing reads. (e) "Base Quality" category uses base-quality information generated by sequencing machine to detect whether low-quality bases are significantly biased to editing-sites. Two attributes, PV2 and BQB belongs to this category. (f) Finally, "Read

Table 2 Attributes used to train prediction model: total 15-features are calculated with samtools-bcftools(v1.2) pipeline [23]

\begin{tabular}{|c|c|c|}
\hline Categories & Attributes & Description \\
\hline Read depth & Read depth & Read depth \\
\hline Allele segregation & VAF & Variant read ratio \\
\hline Allele segregation & SGB & Segregation based metric \\
\hline Allele segregation & $\mathrm{FQ}$ & $\begin{array}{l}\text { Phred probability of all samples } \\
\text { being the same }\end{array}$ \\
\hline Allele segregation & CallQual & Variant/reference QUALity \\
\hline Mapping quality & PV3 & Mapping quality bias \\
\hline Mapping quality & MQB & $\begin{array}{l}\text { Mann-Whitney U test of Mapping } \\
\text { Quality Bias }\end{array}$ \\
\hline Mapping quality & MQOF & Fraction of MQ0 reads \\
\hline Mapping quality & MQ & $\begin{array}{l}\text { Root-mean-square mapping quality } \\
\text { of covering reads }\end{array}$ \\
\hline \multirow[t]{2}{*}{ Read position } & \multirow[t]{2}{*}{ VDB } & $\begin{array}{l}\text { Variant Distance Bias for filtering } \\
\text { splice-site artefacts in }\end{array}$ \\
\hline & & RNA-seq data \\
\hline Read position & $\mathrm{RPB}$ & $\begin{array}{l}\text { Mann-Whitney } U \text { test of Read } \\
\text { Position Bias }\end{array}$ \\
\hline Read position & PV4 & Tail distance bias \\
\hline Base quality & PV2 & Base quality bias \\
\hline Base quality & $\mathrm{BQB}$ & $\begin{array}{l}\text { Mann-Whitney U test of Base } \\
\text { Quality Bias }\end{array}$ \\
\hline Read strand & PV1 & Read strand bias \\
\hline
\end{tabular}


Table 3 Comparison results from two different tissues which shows that RDD occurs condition specifically

\begin{tabular}{lllll}
\hline & Reads & Bases & Raw RDDs & Accepted RDDs \\
\hline Bahn's & $115,132,348$ & $13,815,881,760$ & $6,856,440$ & 105,564 \\
Peng's & $583,640,030$ & $101,787,059,720$ & $58,666,976$ & $3,076,908$ \\
Fold & 5.07 & 7.37 & 8.56 & 29.15 \\
\hline
\end{tabular}

Strand" category includes single attribute PV1, that represents how the strands of edited reads are biased than non-edited reads (Table 2).

\section{Results}

\section{Evaluation with two previous studies}

We evaluated RDDpred with two datasets from independent studies performed by Bahn's and Peng's group, respectively $[6,7]$. Both studies computationally predicted RNA-editing sites and validated them with Sanger-seq. In Bahn's study, RNA-seq produced 115,132,348 reads with $13,815,881,760$ bases in human glioblastoma astrocytoma. RDDpred detected 6,856,440 raw RDDs from them and predicted 105,564 sites as true RNA-editings. In Peng's study, RNA-seq produced 583,640,030 reads with $101,787,059,720$ bases in human lymphoblastoid. In this case, RDDpred detected 58,666,976 raw RDDs from them and predicted 3,076,908 sites as true RNA-editings. Note that even though both study uses human tissues, they resulted different number of RNA-editing sites, 105,564 vs. 3,076,908, which indicates that the expression patterns of RNA-editing events might be different in two experimental conditions (Table 3 ).

We constructed condition-specific model independently from each datasets and evaluated RDDpred separately with the corresponding validation results as test-data. And the test-data was the results of Sanger-seq by each groups. In the process of constructing each models, we carefully arranged the training-data in order not to contain any of information related to test-data. In other words, we did not use test-data for the construction of the model.

1. Training datasets

(a) Positive examples: Predicted as positives sites by Public Databases (RADAR, DARNED)

(b) Negative examples: Predicted as artefact sites by MES method (Peng et al. Nature biotechnology 2012)

(c) The entries overlapped with test-data are excluded from training-data

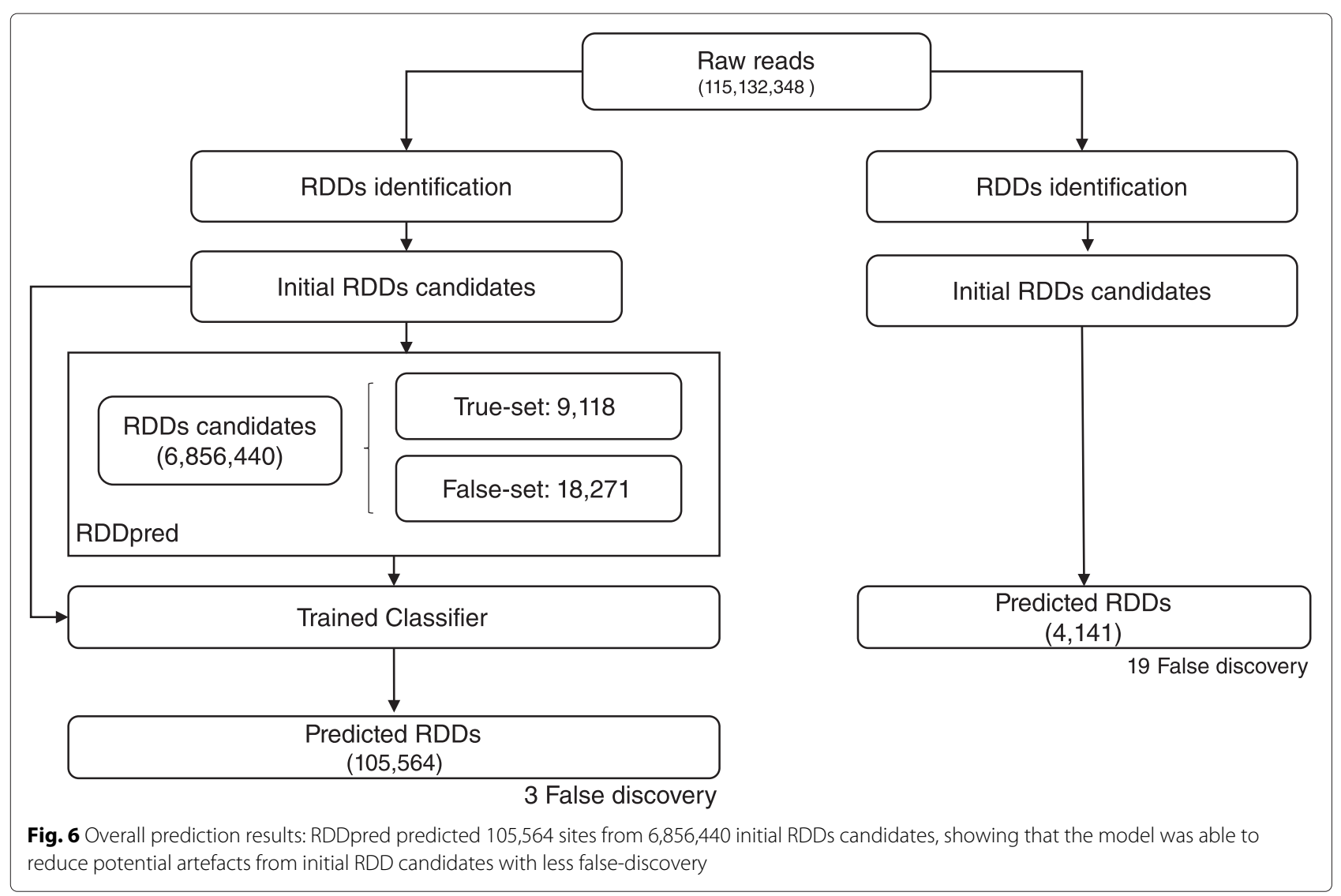




\section{Test datasets}

(a) Positive examples: Positively detected sites by experimental validation (Sanger-seq)

(b) Negative examples: False discovery sites proved by experimental validation (Sanger-seq)

\section{RDDpred prediction in Bahn's dataset}

RDDpred predicted 105,564 sites from 6,856,440 initial RDDs candidates, showing that not only the model was able to reproduce $(95.32 \%)$ the results but also was able to reduce potential artefacts (98.46 \%) from Bahn's study. Moreover, RDDpred have successfully reject most of the false discoveries in Bahn's prediction. Resulting $84.21 \%$ NPV(Negative Predictive Value). The following results indicate that RDDpred showed relative robustness than Bahn's by reducing potential artefacts and rejecting false-discoveries (Figs. 6 and 7).

\section{RDDpred prediction in Peng's dataset}

In Peng's study, RDDpred predicted 3,076,908 sites from $58,666,976$ initial RDDs candidates, which also proves that the model was able to successfully reproduce $(90.37 \%)$ the results but also reduce potential artefacts (94.79\%) from Peng's study. Moreover, RDDpred have successfully reject most of the false discoveries in Peng's prediction. Resulting 75.86 \% NPV (Negative Predictive Value). The following results indicate that RDDpred also showed relative robustness than Peng's by reducing potential artefacts and rejecting false-discoveries (Figs. 8 and 9).

\section{Additional specification of RDDpred}

To provide information about actual running-time and memory-usage of RDDpred, we monitored the resourceusage while processing the Peng's dataset. With the machine specified below, RDDpred took 18.33 hours to process $583,640,030$ reads of $101,787,059,720$ bases. We believe that the resources used here are not too excessive for common research groups.

1. Linux version: Linux version 2.6.32-358.el6.x86_64, CentOS release 6.4

2. Memory usage: $20 \mathrm{~GB}$ in maximum

3. CPU usage: 20-cores (Intel(R) Xeon(R) CPU E5645 @ $2.40 \mathrm{GHz})$

\section{Discussion}

We developed a software package for RNA-editing prediction from RNA-seq data. RDDpred utilizes current

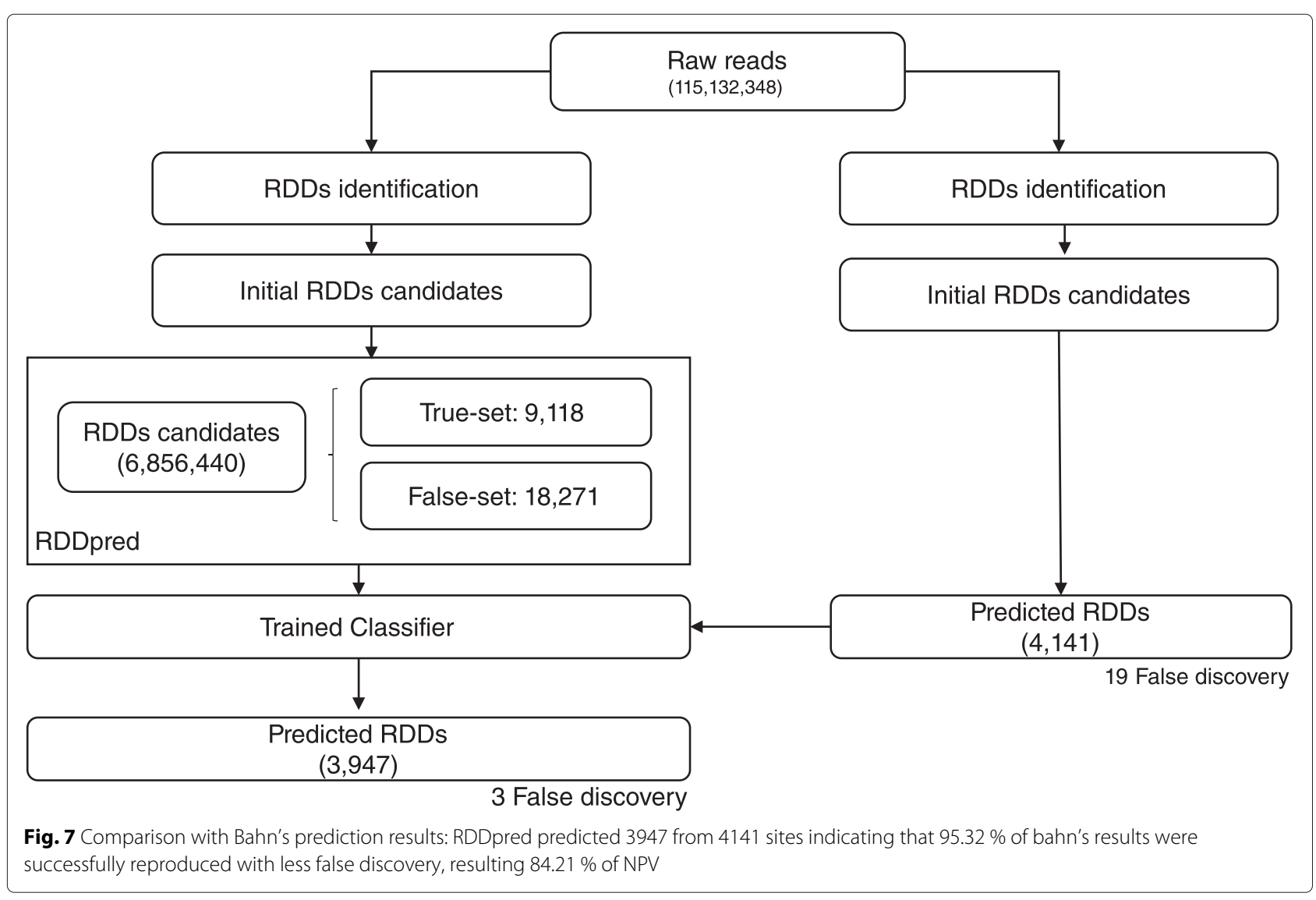




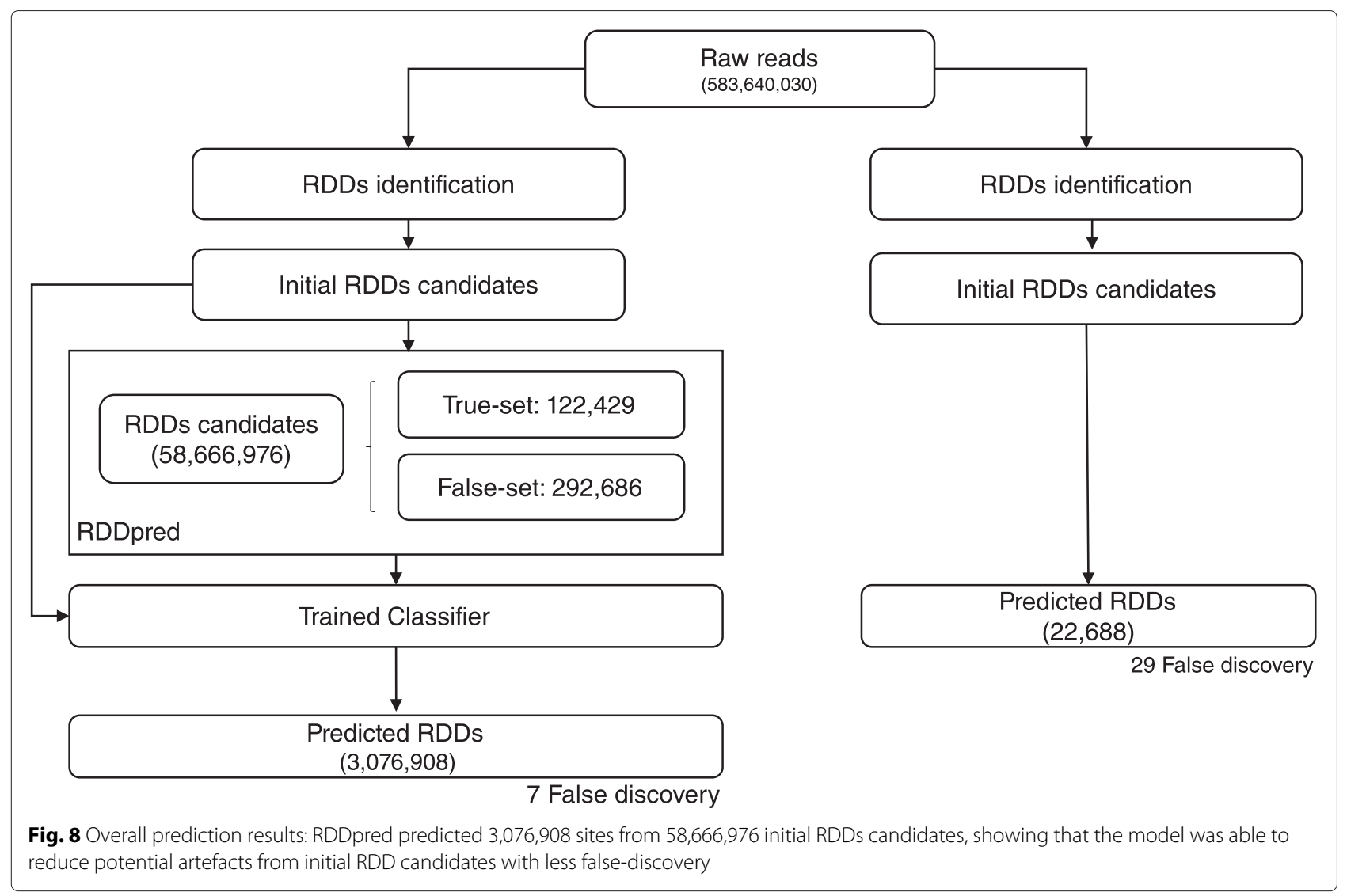

published database and methods such as RADAR, DARNED [13, 29] and MES-method [6] to build condition specific predictor. RDDpred generates a predictor that considers the experimental condition under which RNAseq experiments are performed. As of now, there are only two studies we can compare with RDDpred. However, we successfully demonstrated that RDDpred was able to reproduce the results and reduce the false-discovery in both studies.

In order to investigate working principle of RDDpred in terms of prediction power of each categories, we calculated information-gain values with WEKA [30]. The rankings of six categories are listed in Table 4. Top 3 categories ("Read Depth", "Allele Segregation", and "Base Quality") have strong prediction power. It is well known that "Read depth" or "Allele Segregation" categories of features are generally used for assessing the authenticity of variants [5, 7]. However, we discovered that the "Base Quality" category of attributes might also have significant prediction power according to the prediction results of two datasets.

During the high-throughput sequencing, the sequencers generate bases-qualities that represent the confidence of sequencing. Therefore, unlike other five metrics, the "Base Quality" reflects the molecular status of bases that are directly recorded by sequencer. Until now, we only knew bases modified by editing enzymes are somehow recognized as guanine (or thymine for APOBEC class), but did not know how these recognitions are observed in the perspective of sequencing machines. The base-quality issue indicates that there might be some distinctions between normal and edited bases at the molecular level. Thus, it implies that more detailed recording of molecular characteristics during sequencing process might be a key to improve the accuracy of RNA-editing detection.

\section{Conclusions \\ RDDpred: a useful tool for investigating condition-specific RNA-editing with RNA-seq}

RNA-seq is one of the most powerful methods to investigate transcriptome and the amount of RNA-seq has recently increased nearly exponentially [31]. In spite of this rapid RNA-seq data accumulation and the recognition on important biological roles of RNA-editing, only a few studies reported RNA-editing findings due to the difficulty of getting robust profiles of RNA-editome [16]. Since it is difficult to perform the experimental validation of RNA-editing events in whole-transcriptome scale, a reliable and easily-usable prediction method is truly required. 


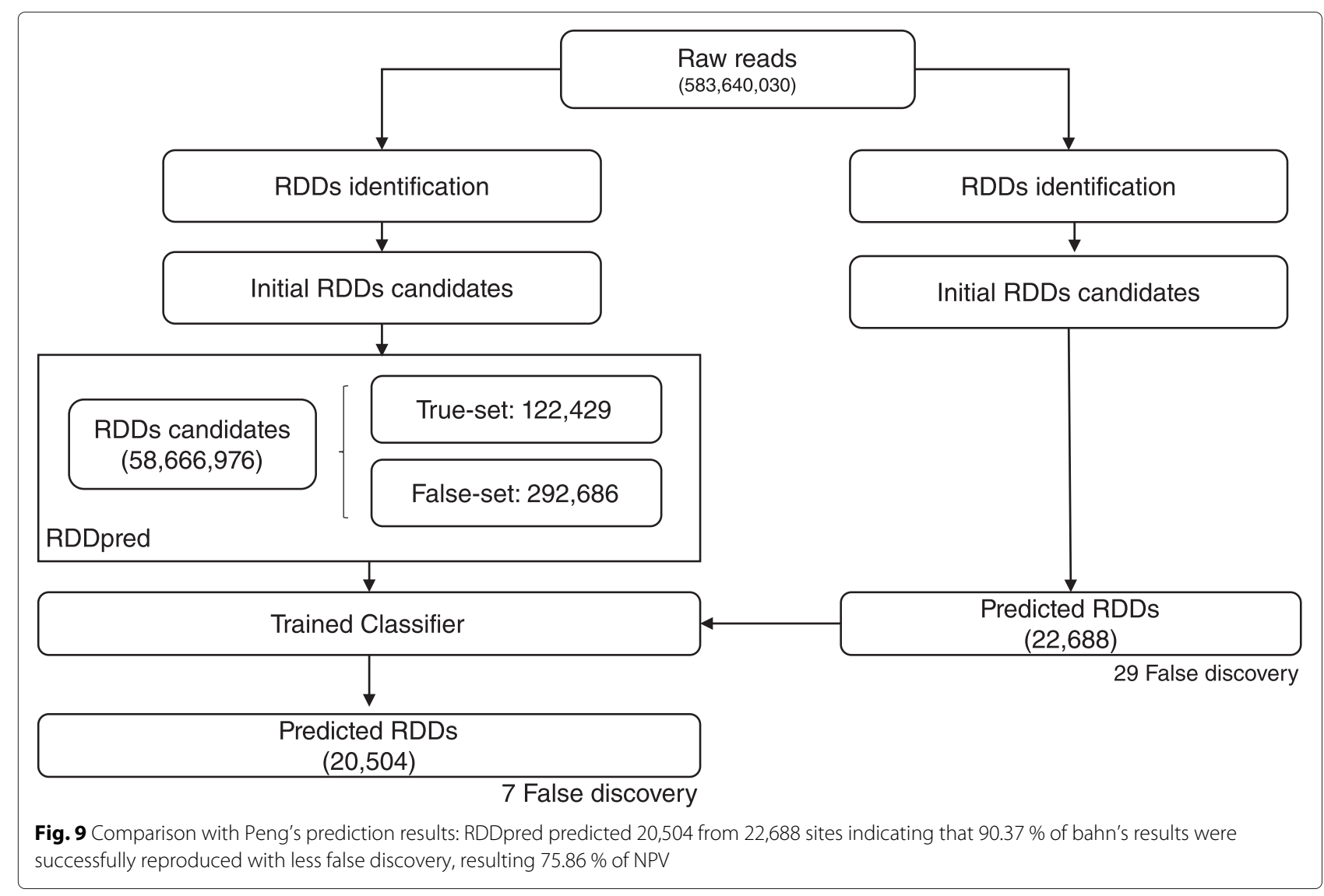

RDDpred prepares training examples that are specific to the condition of input data without experimental validations. RDDpred proved good performances by reproducing the detection of two previous studies and correcting most of their false-discoveries. Moreover, as far as we know, RDDpred is the very first automated pipeline that utilizes machine-learning technique with a wellevaluated performance. Thus, we believe that RDDpred will be very useful and can contribute significantly to the

Table 4 Category rankings of attributes utilized by RDDpred model: top 3 categories showed relatively strong prediction power

\begin{tabular}{llllll}
\hline Category & DataA & DataB & RankA & RankB & RankMean \\
\hline Read depth & 0.4943 & 0.2515 & 1 & 2 & 1.5 \\
Base quality & 0.4498 & 0.40195 & 3 & 1 & 2 \\
$\begin{array}{l}\text { Allele } \\
\text { segregation }\end{array}$ & 0.457975 & 0.222825 & 2 & 3 & 2.5 \\
$\begin{array}{l}\text { Read } \\
\text { position }\end{array}$ & 0.195767 & 0.1019 & 4 & 4 & 4 \\
$\begin{array}{l}\text { Mapping } \\
\text { quality }\end{array}$ & 0.010025 & 0.047 & 6 & 5 & 5.5 \\
Read strand & 0.1584 & 0.0216 & 5 & 6 & 5.5 \\
\hline
\end{tabular}

study of RNA-editing. RDDpred is available at http : / / biohealth.snu.ac.kr/software/RDDpred.

\section{Competing interests}

The authors declare that they have no competing interests.

\section{Authors' contributions}

SK designed and supervised the research project and edited the paper. MK developed the method, performed data analysis and wrote the manuscript. $\mathrm{BH}$ helped to make the website and advised and assisted the manuscript. All authors read and approved the final manuscript.

\section{Declarations}

This article has been published as part of BMC Genomics Volume 17 Supplement 1, 2016: Selected articles from the Fourteenth Asia Pacific Bioinformatics Conference (APBC 2016): Genomics. The full contents of the supplement are available online at http://www.biomedcentral.com/ bmcgenomics/supplements/17/S1.

\section{Acknowledgements}

This research was supported by the Bio \& Medical Technology Development Program of the NRF funded by the Korean government, MSIP(No. NRF-2014M3C9A3063541). Also, this research was supported by Next-Generation Information Computing Development Program through the National Research Foundation of Korea(NRF) funded by the Ministry of Science, ICT \& Future Planning (No. NRF-2012M3C4A7033341). The publication cost will be paid by the Seoul National University Office of Research.

\section{Author details}

${ }^{1}$ Interdisciplinary Program in Bioinformatics, Seoul National University, Seoul, Republic of Korea. ${ }^{2}$ Department of Computer Science and Engineering, Seoul 
National University, Seoul, Republic of Korea. ${ }^{3}$ Bioinformatics Institute, Seoul National University, Seoul, Republic of Korea.

\section{Published: 11 January 2016}

\section{References}

1. Burns CM, Chu H, Rueter SM, Hutchinson LK, Canton H, Sanders-Bush E, et al. Regulation of serotonin-2c receptor g-protein coupling by rna editing. Nature. 1997;387:303-8.

2. Keegan LP, Leroy A, Sproul D, O'Connell MA. Adenosine deaminases acting on rna (adars): Rna-editing enzymes. Genome Biol. 2004;5:209.

3. Harris RS, Petersen-Mahrt SK, Neuberger MS. Rna editing enzyme apobec 1 and some of its homologs can act as dna mutators. Mol cell. 2002;10:1247-53.

4. Nishikura K. Functions and regulation of rna editing by adar deaminases. Ann Rev Biochem. 2010;79:321.

5. St Laurent G, Tackett MR, Nechkin S, Shtokalo D, Antonets D, Savva YA, et al. Genome-wide analysis of a-to-i rna editing by single-molecule sequencing in drosophila. Nat Struct Mol Biol. 2013;20:1333-39.

6. Peng Z, Cheng Y, Tan BC-M, Kang L, Tian Z, Zhu Y, et al. Comprehensive analysis of rna-seq data reveals extensive rna editing in a human transcriptome. Nat Biotechnol. 2012;30:253-60.

7. Bahn JH, Lee JH, Li G, Greer C, Peng G, Xiao X. Accurate identification of a-to-i rna editing in human by transcriptome sequencing. Genome Res. 2012;22:142-50.

8. Rueter SM, Dawson TR, Emeson RB. Regulation of alternative splicing by rna editing. Nature. 1999;399:75-80.

9. Rosenberg BR, Hamilton CE, Mwangi MM, Dewell S, Papavasiliou FN. Transcriptome-wide sequencing reveals numerous apobec1 mrna-editing targets in transcript 3' utrs. Nat Struct Mol Biol. 2011;18:230-6.

10. Nishikura K. Editor meets silencer: crosstalk between rna editing and rna interference. Nat Rev Mol Cell Biol. 2006;7:919-31.

11. Galeano F, Rossetti C, Tomaselli S, Cifaldi L, Lezzerini M, Pezzullo M, et al. Adar2-editing activity inhibits glioblastoma growth through the modulation of the cdc14b/skp2/p21/p27 axis. Oncogene. 2013;32: 998-1009.

12. Chiu YL, Soros VB, Kreisberg JF, Stopak K, Yonemoto W, Greene WC. Cellular apobec $3 \mathrm{~g}$ restricts hiv-1 infection in resting cd4+t cells. Nature. 2010;466:276-6.

13. Kiran A, Baranov PV. Darned: a database of rna editing in humans. Bioinformatics. 2010;26:1772-6.

14. Song W, Liu Z, Tan J, Nomura Y, Dong K. Rna editing generates tissue-specific sodium channels with distinct gating properties. J Biol Chem. 2004;279:32554-2561.

15. Miyata $Y$, Sugita M. Tissue-and stage-specific rna editing of rps14 transcripts in moss (physcomitrella patens) chloroplasts. J Plant Physiol. 2004; 161:113-5.

16. Bass B, Hundley H, Li JB, Peng Z, Pickrell J, Xiao XG, et al. The difficult calls in rna editing. Nat Biotechnol. 2012;30:1207-9.

17. Degner JF, Marioni JC, Pai AA, Pickrell JK, Nkadori E, Gilad Y, et al. Effect of read-mapping biases on detecting allele-specific expression from rna-sequencing data. Bioinformatics. 2009;25:3207-212.

18. Heap GA, Yang JH, Downes K, Healy BC, Hunt KA, Bockett N, et al. Genome-wide analysis of allelic expression imbalance in human primary cells by high-throughput transcriptome resequencing. Hum Mol Genet. 2010;19:122-34.

19. Talwalkar A, Liptrap J, Newcomb J, Hartl C, Terhorst J, Curtis K, et al. Smash: a benchmarking toolkit for human genome variant calling. Bioinformatics. 2014;30:2787-95.

20. Engström PG, Steijger T, Sipos B, Grant GR, Kahles A, Rätsch G, et al. Systematic evaluation of spliced alignment programs for rna-seq data. Nat Methods. 2013;10:1185-91.

21. Dobin A, Davis CA, Schlesinger F, Drenkow J, Zaleski C, Jha S, et al. Star: ultrafast universal rna-seq aligner. Bioinformatics. 2013;29:15-21.

22. Pruitt KD, Brown GR, Hiatt SM, Thibaud-Nissen F, Astashyn A, Ermolaeva $O$, et al. Refseq: an update on mammalian reference sequences. Nucleic Acids Res. 2014;42:756-63.

23. Li H. A statistical framework for snp calling, mutation discovery, association mapping and population genetical parameter estimation from sequencing data. Bioinformatics. 2011;27:2987-993.
24. McKenna A, Hanna M, Banks E, Sivachenko A, Cibulskis K, Kernytsky A et al. The genome analysis toolkit: a mapreduce framework for analyzing next-generation dna sequencing data. Genome Res. 2010;20:1297-303.

25. Karolchik D, Hinrichs AS, Furey TS, Roskin KM, Sugnet CW, Haussler D, et al. The ucsc table browser data retrieval tool. Nucleic Acids Res. 2004;32:493-6.

26. Li JB, Levanon EY, Yoon JK, Aach J, Xie B, LeProust E, et al. Genome-wide identification of human rna editing sites by parallel dna capturing and sequencing. Science. 2009;324:1210-13.

27. Mo F, Wyatt AW, Sun Y, Brahmbhatt S, McConeghy BJ, Wu C, et al. Systematic identification and characterization of rna editing in prostate tumors. PloS One. 2014;9(7):e101431.

28. Zhang Q, Xiao X. Genome sequence-independent identification of rna editing sites. Nat Methods. 2015;12:347-50.

29. Ramaswami G, Li JB. Radar: a rigorously annotated database of a-to-i rna editing. Nucleic Acids Res. 2014;42(D1):D109-D113.

30. Hall M, FrankE, Holmes G, Pfahringer B, Reutemann P, Witten IH. The weka data mining software: an update. ACM SIGKDD explorations newsletter. 2009;11:10-18.

31. Leinonen $R$, Sugawara $H$, Shumway $M$. The sequence read archive. Nucleic Acids Res. 2011;39(suppl 1):D19-D21.

\section{Submit your next manuscript to BioMed Central and we will help you at every step:}

- We accept pre-submission inquiries

- Our selector tool helps you to find the most relevant journal

- We provide round the clock customer support

- Convenient online submission

- Thorough peer review

- Inclusion in PubMed and all major indexing services

- Maximum visibility for your research

Submit your manuscript at www.biomedcentral.com/submit 